\title{
Bulk electronic structure of high-order quaternary approximants
}

\author{
Shuvam Sarkar $\odot,{ }^{1}$ Pampa Sadhukhan, ${ }^{1}$ Vipin Kumar Singh $\odot,{ }^{1}$ Andrei Gloskovskii, ${ }^{2}$ Kazuhiko Deguchi, ${ }^{3}$ \\ Nobuhisa Fujita $\odot,{ }^{4}$ and Sudipta Roy Barman $\odot^{1}$ \\ ${ }^{1}$ UGC-DAE Consortium for Scientific Research, Khandwa Road, Indore 452001, Madhya Pradesh, India \\ ${ }^{2}$ Deutsches Elektronen-Synchrotron DESY, Notkestr. 85, D-22607 Hamburg, Germany \\ ${ }^{3}$ Department of Physics, Graduate School of Science, Nagoya University, Nagoya 464-8602, Japan \\ ${ }^{4}$ Institute of Multidisciplinary Research for Advanced Materials, Tohoku University, Sendai 980-8577, Japan
}

(Received 30 June 2020; accepted 21 January 2021; published 16 February 2021)

\begin{abstract}
The bulk electronic structures of two high-order quaternary approximants of F-type icosahedral (i)-Al-PdTM quasicrystal: Al-Pd-Cr-Fe and Al-Pd-Mo-Fe, having similar electron to atom $(e / a)$ ratio as $i$-Al-Pd-Mn quasicrystal, have been investigated by hard x-ray photoelectron spectroscopy. We establish the presence of a well-formed pseudogap at the Fermi level in both the approximants. The pseudogap turns out to be deeper in the approximants compared to $i$-Al-Pd-Mn, and this is supported by specific heat data. Modifications in the line shape of $\mathrm{Al} 2 s$ core-level main peak as well as the plasmon loss peaks indicate enhanced hybridization of $\mathrm{Al} s p$ and transition metal $d$ states in the approximants, which could be one of the possible reasons for their pseudogap. The absence of magnetic exchange splitting in the Fe $2 p$ core-level spectra establishes the nonmagnetic nature of the approximants.
\end{abstract}

DOI: 10.1103/PhysRevResearch.3.013151

\section{INTRODUCTION}

Approximants, the crystalline analogues of quasicrystals, are periodic systems with translational symmetry [1]. They connect ordinary crystals and quasicrystals, where the latter do not exhibit translational symmetry but possess an aperiodic long-range order with forbidden rotational symmetries [2]. Quasicrystals and related approximants have been observed in metallic alloys $[1,3,4]$, oxide thin films [5,6], binary nanoparticle systems [7], elemental metallic clathrate layers [8], and soft matter [9]. Interesting developments in this area are the observation of unconventional quantum critical phenomenon [10], appearance of superconductivity [11,12], prediction of nontrivial topological edge states [13-15], evidence of ferromagnetic [16] and antiferromagnetic ordering [17,18], identification of surface resonance states [19], and demonstration of dodecagonal approximants in coordination polymers [20], to name a few.

An approximant usually has a large unit cell accommodating many atoms, whose arrangement is considered to mimic the local atomic structure of a quasicrystal. Both the approximant and the related quasicrystalline phase can be described as lower dimensional projections of the same hypercrystal along rational and irrational directions, respectively [21]. In this work, we restrict our attention to the approximants of icosahedral quasicrystals, and so the word

Published by the American Physical Society under the terms of the Creative Commons Attribution 4.0 International license. Further distribution of this work must maintain attribution to the author(s) and the published article's title, journal citation, and DOI. "approximants" will henceforth be used for this particular class. Many approximants have so far been successfully synthesized and their structural and transport properties have been studied [1,21-27]. The intriguing transport properties of icosahedral quasicrystals such as high resistivity and its negative temperature coefficient are also observed in highorder approximants that have large sized unit cells with more than $\sim 500$ atoms $[23,24,26]$. In contrast, low-order approximants with smaller unit cells often exhibit metallic behavior $[23,24,26,28]$.

The importance of approximants in quasicrystal research is evident from the fact that density functional theory (DFT) calculations have been performed exclusively for approximants [29-35], where higher order approximants are assumed to represent the quasicrystalline phase. Approximants as well as icosahedral quasicrystals are often described as HumeRothery type electron compounds, in which the structure is stabilized at certain $e / a$ ratio (ratio of the number of valence electrons to the number of atoms) [36]. In a nearly-free electron scenario, the Hume-Rothery mechanism produces a dip or pseudogap in the density of states at the Fermi level $\left(E_{F}\right)$ as a consequence of the Fermi surface- quasi-Brillouin zone (BZ) interaction, provided $|\mathbf{G}| \approx 2 k_{F}$ is satisfied, where $\mathbf{G}$ represents the reciprocal lattice vectors that generate strong Bragg diffractions and $k_{F}$ is the Fermi radius. In other words, an energetic stability is provided if $E_{F}$ lies within the pseudogap, which requires a specific value of $e / a$. The presence of the pseudogap was established by $a b$ initio electronic structure calculations for a series of approximants representing icosahedral (i)-Al-Pd-Mn [37] with increasing size of the unit cell [32]. The pseudogaps were less prominent in loworder approximants $(1 / 1$, and $2 / 1)$ of $i$-Al-Pd-Mn, whereas in 
high-order approximants $(5 / 3$ and $8 / 5)$ well-formed pseudogaps were found to occur at $E_{F}$ [32].

In spite of extensive structural, transport and theoretical studies, experimental investigations on the bulk electronic structure of approximants using photoelectron spectroscopy have been rarely performed to date. In fact, low-temperature ultrahigh-resolution photoemission spectroscopy showed that the electron density of states near $E_{F}$ were suppressed in the quasicrystalline phase, $\mathrm{Cd}_{5.7} \mathrm{Yb}$, compared to the lowest-order $1 / 1$ approximant, $\mathrm{Cd}_{6} \mathrm{Yb}$ [38]. However, the deeper pseudogap in the quasicrystal was attributed to a stronger hybridization of the $\mathrm{Cd} 5 p$ and $\mathrm{Yb} 5 d$ states in the quasiperiodic environment as compared to the case of $1 / 1$ approximant [39]. Besides, in spite of the predictions by theory, none of the surface-sensitive-low-energy photoemission studies could clearly identify the pseudogap in the electronic structure of approximants. This is possibly because the surface composition and the surface electronic structure could be substantially different from the bulk, as reported for $i$-Al-Pd-Mn [40,41].

Using electron-energy-loss spectroscopy that is a bulk sensitive probe, the plasmon line width in the metastable quasicrystalline phase of $\mathrm{Al}_{6} \mathrm{Mn}$ was reported to be larger than the crystalline phase [42]. Later, the enhancement was found to be much stronger in the thermodynamically stable $i-\mathrm{Al}_{65} \mathrm{Cu}_{20} \mathrm{Ru}_{15}$ quasicrystal [43], possibly indicating strong hybridization of $\mathrm{Al} s p$ and transition metal $d$ states in quasicrystals. Electron density determined from synchrotron radiation powder x-ray diffraction related the origin of the pseudogap in $\alpha$-Al-Mn-Si $1 / 1$ approximant to occurrence of covalent bonds [44].

Al-Pd-Cr-Fe and Al-Pd-Mo-Fe are two high-order approximants in Al-based alloys that have been discovered recently $[45,46]$, and it has not been clarified what in the formation process differentiates these high-order approximant structures from quasicrystals. An advantage of studying the electronic structure of high-order approximants vis-a-vis loworder approximants is that because of the large unit cell of the former [45], a sizable volume of the real space mimics the quasiperiodic order rendering these more quasicrystallike. More specifically, Al-Pd-Cr-Fe and Al-Pd-Mo-Fe have unit cell size of $40.5 \AA$ with 4320 atoms/unit cell, containing 264 interpenetrating atomic clusters, 128 of which are pseudo-Mackay type and the others are mini-Bergman type structures. Although the atomic structure of $i$-Al-Pd-Mn has not been solved, it most likely comprises similar kinds of clusters $[47,48]$.

In recent years, hard x-ray photoemission spectroscopy (HAXPES) has turned out to be one of the most important techniques in providing direct information about the bulk electronic structure of materials owing to its large probing depth [49-54]. HAXPES established the existence of the pseudogap in a series of icosahedral quasicrystals such as $i$-Al-Pd-Mn, $i$-Al-Cu-Fe, $i-\mathrm{Zn}-\mathrm{Mg}-\mathrm{Y}$, and $i$-Zn-Mg-Dy [55,56]. The purpose of the present work is to investigate the bulk electronic structure of high-order approximants Al-Pd-Cr-Fe and Al-Pd-Mo-Fe using HAXPES, and with particular focus on whether the pseudogap exists, and if so, what is its nature in comparison to a closely related icosahedral quasicrystal (i-Al-Pd-Mn) [37].

\section{EXPERIMENTAL METHOD}

The HAXPES measurements were carried out at the P09 beamline in Petra-III, DESY with the sample held at $50 \mathrm{~K}$ [57]. A photon energy $(h v)$ of $5.95(\approx 6) \mathrm{keV}$ was used, with the corresponding inelastic mean free path being about $85 \AA$ A. A post-monochromator was used to improve the resolution and stability of the photon beam [57]. The photons were incident at a nearly grazing angle and the measurements were performed in the normal emission geometry, with the electron energy analyzer having an angular acceptance angle of $\pm 15^{\circ}$, see Ref. [58] for the details of the experimental setup. The samples were fractured in ultra-high vacuum in order to remove the thick native oxide layers present on the surface. The base pressure of the chamber was $3 \times 10^{-10}$ mbar. The Fermi level $\left(E_{F}\right)$ position as well as the total energy resolution $(300 \mathrm{meV})$, which includes both the analyzer and photon source broadening, was obtained by least-squares fitting of the Fermi edge of a gold foil in electrical contact with the specimen, and its position is taken as the zero of the binding energy (BE) scale.

Single-phase polycrystalline Al-Pd-Cr-Fe and Al-Pd-MoFe were obtained by annealing arc-melted ingots under Argon atmosphere at $1123 \mathrm{~K}$ (6 days) and $1173 \mathrm{~K}$ (4 days), respectively. The compositions were $\mathrm{Al}_{69.4} \mathrm{Pd}_{22.3} \mathrm{Cr}_{2.2} \mathrm{Fe}_{6.1}$ (Al-Pd$\mathrm{Cr}-\mathrm{Fe}$ ) [45] and $\mathrm{Al}_{70.8} \mathrm{Pd}_{20.3} \mathrm{Mo}_{0.9} \mathrm{Fe}_{7.9}$ (Al-Pd-Mo-Fe) [46], as previously estimated for the two approximants using electron probe micro-analyzer (EPMA). High-resolution powder $\mathrm{x}$-ray diffraction (Fig. S1 in Ref. [59]) has confirmed that both the samples are single phase without any sign of impurities. Although the crystal structure analysis of Al-Pd-Mo-Fe is still under way, Fig. S1 also shows that it is isostructural to Al$\mathrm{Pd}-\mathrm{Cr}-\mathrm{Fe}$, which has cubic space group $P a \overline{3}$ (No. 205), with a large unit cell comprising of about 4320 atoms [45]. Single grain $i$ - $\mathrm{Al}_{70.5} \mathrm{Pd}_{21} \mathrm{Mn}_{8.5}$ (i-Al-Pd-Mn) used for HAXPES was prepared using the Czochralski method [60]. Specific heat was measured down to $1.8 \mathrm{~K}$ with a Quantum Design PPMS by a thermal relaxation method.

The core-level main peaks have been fitted by using Doniach- $\breve{S}$ unjić (DS) line shape [61] and asymmetric Lorentzian line shapes have been used to represent the plasmon loss peaks [62]. The lifetime broadenings of the core level main peak, the DS asymmetry parameter $(\alpha)$, intensities, peak positions, and the background [63] are varied independently, as in our earlier work [64]. Recoil effect in HAXPES shifts the core-level peaks to higher BE in light materials [65]. However, the recoil effect was not observed in $i$-Al-Pd-Mn since it involves heavier $d$ metals and this is evident from the valence band as well as $\mathrm{Al} 2 s$ spectra [55]. Since in $\mathrm{Al}-\mathrm{Pd}-\mathrm{Cr}-\mathrm{Fe}$ and $\mathrm{Al}-\mathrm{Pd}-\mathrm{Mo}-\mathrm{Fe}$, atoms of larger atomic weight $(\mathrm{Fe}, \mathrm{Mo})$ mostly replace $\mathrm{Mn}$ in $i$-Al-Pd-Mn, the recoil effect is also not observed in the approximants.

\section{RESULTS}

The HAXPES valence band (VB) spectra of Al-Pd-Cr-Fe, Al-Pd-Mo-Fe, and $i$-Al-Pd-Mn [Fig. 1(a)] show a prominent peak around $4.4 \mathrm{eV}$ that can be assigned to the $\mathrm{Pd}$ $4 d$ states $[32,55,66]$. It appears at similar binding energy in both the approximants in comparison to $i$-Al-Pd-Mn that 


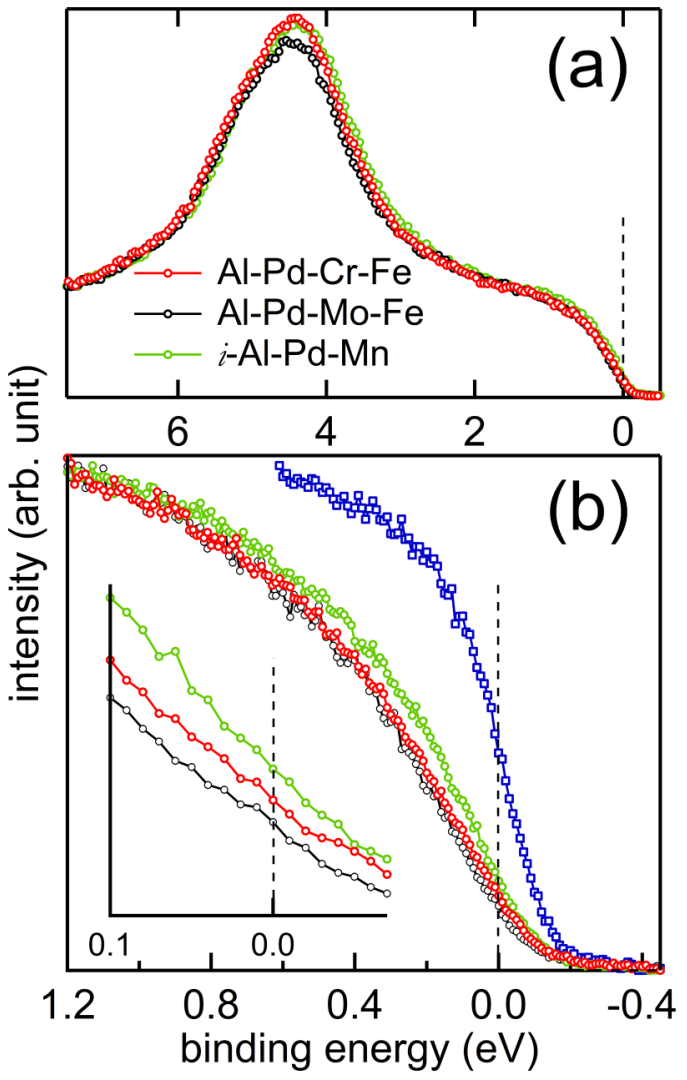

FIG. 1. (a) The HAXPES valence band (VB) spectra of Al-Pd$\mathrm{Cr}-\mathrm{Fe}$ and Al-Pd-Mo-Fe compared with $i$-Al-Pd-Mn. All the spectra have been taken with $h v=6 \mathrm{keV}$ at $50 \mathrm{~K}$ and have been normalized in the background region at around $7.5 \mathrm{eV}$ binding energy. (b) The near $E_{F}$ spectral region of Al-Pd-Cr-Fe, Al-Pd-Mo-Fe, and $i$-Al-Pd-Mn recorded with smaller step size and better signal to noise ratio along with a metallic Fermi edge from a gold foil (blue open squares), the inset shows the region around $E_{F}$ in an expanded scale.

does not show recoil effect [55], indicating the absence of recoil effect in the former. It is slightly reduced in intensity for Al-Pd-Mo-Fe, the reason for which will be discussed latter. The differences in the spectral shape in the near $E_{F}$ region is evident from Fig. 1(b). An interesting observation is that both Al-Pd-Mo-Fe and Al-Pd-Cr-Fe exhibit a spectral shape characteristic of a pseudogap [55,56], which is starkly different from the metallic Fermi edge. In fact, the spectral intensity is more suppressed in both the approximants compared to $i$-Al-Pd-Mn and this trend continues up to $E_{F}$ [inset, Fig. 1(b)]. This spectral shape indicates the existence of the pseudogap in both the approximants, which is possibly deeper compared to $i$-Al-Pd-Mn.

In order to extract the shape of the pseudogap quantitatively, we have performed a least-squares fitting of the near $\mathrm{E}_{F}$ region using the expression

$$
[I \times S(E) \times f(E, T)] \otimes G(E),
$$

where $f(E, T)$ is the Fermi function, $I$ is a multiplicative factor, and the Gaussian function $G(E)$ represents the instrumental resolution that is kept fixed. $S(E)$ represents the pseudogap defined as a minimum in the density of states. It is represented by an inverted Lorentzian function (iLf) as
TABLE I. The parameters (and their standard deviations, see text) obtained from the least-squares curve fitting of the near $\mathrm{E}_{F}$ spectra of $i$-Al-Pd-Mn, Al-Pd-Cr-Fe, and Al-Pd-Mo-Fe using an inverted Lorentzian function (iLf) with straight line.

\begin{tabular}{lcccc}
\hline \hline & \multicolumn{5}{c}{ Inverted Lorentzian with straight line } \\
\cline { 2 - 5 } Specimen & $\mathrm{C}_{L}$ & $2 \Gamma_{L}$ & $a$ & $b$ \\
\hline$i$-Al-Pd-Mn & $0.63 \pm 0.02[69]$ & $0.53 \pm 0.13$ & $0.72 \pm 0.04$ & $0.23 \pm 0.04$ \\
Al-Pd-Cr-Fe & $0.73 \pm 0.02$ & $0.51 \pm 0.15$ & $0.65 \pm 0.04$ & $0.27 \pm 0.04$ \\
Al-Pd-Mo-Fe & $0.79 \pm 0.02$ & $0.57 \pm 0.15$ & $0.71 \pm 0.07$ & $0.23 \pm 0.07$ \\
\hline
\end{tabular}

$S(E)=(a+b E) \times\left[1-\frac{C_{L} \Gamma_{L}^{2}}{E^{2}+\Gamma_{L}^{2}}\right]$, where $C_{L}$ indicates the depth of the pseudogap and $2 \Gamma_{L}$ is the FWHM [55,56,67,68]. For $C_{L}=1$, the pseudogap is fully formed with zero spectral intensity at the minimum. On the other hand, for $C_{L}=0$, the pseudogap is absent and $S(E)$ is represented by a straight line $(a+b E)[55,56,67,68]$. The position of the minimum of the pseudogap is kept fixed at $E_{F}$ (zero in the BE scale), as in our previous work [55,56]. $a$ and $b$ in $(a+b E)$ are generally determined from a prior straight line fit in the higher BE region, e.g., $0.7-1.2 \mathrm{eV}[55,67,68]$, and are kept fixed in the subsequent overall fitting with iLf. However, this makes the fitting somewhat dependent on the $\mathrm{BE}$ range chosen for the straight line fit. So, here we find $a$ and $b$ for many different possible $\mathrm{BE}$ ranges (see Tables S1-S3 of Ref. [59]). This provides allowed ranges of variation in $a$ and $b$, and for the final fitting, $a$ and $b$ are varied within this range (free variation of both $a$ and $b$ gives unphysical results). Thus the fitting becomes independent of the range for the linear fitting.

In Fig. 2, presence of the pseudogap in both the high-order approximants is established by the spectral function $S(E)$ obtained from the fitting that shows a deep minimum at $E_{F}$. The quality of the fit is good, as shown by the fitted curve (red) and the scatter of its residual (black curve). We find that the depth of the pseudogap in both the approximants is larger compared to the quasicrystal: $\mathrm{C}_{L}$ for $i-\mathrm{Al}-\mathrm{Pd}-\mathrm{Mn}, \mathrm{Al}-\mathrm{Pd}-\mathrm{Cr}-\mathrm{Fe}$, and $\mathrm{Al}-$ $\mathrm{Pd}-\mathrm{Mo}-\mathrm{Fe}$ are found to be $0.63,0.73$, and 0.79 , respectively, whereas $2 \Gamma_{L}, a$ and $b$ are similar (Table I, and row 16 of Tables S1- S3 of Ref. [59]). Thus the conclusions from the fitting are (i) the existence of a well-formed pseudogap in the high-order approximants Al-Pd-Cr-Fe and Al-Pd-Mo-Fe, (ii) it is deeper compared to $i$-Al-Pd-Mn while their widths are similar, and (iii) the depth of the pseudogap is somewhat larger in $\mathrm{Al}-\mathrm{Pd}-\mathrm{Mo}-\mathrm{Fe}$ as compared to $\mathrm{Al}-\mathrm{Pd}-\mathrm{Cr}-\mathrm{Fe}$. These become evident from Fig. 2(d), where the $S(E)$ of the three compounds are plotted together.

Although the fitting procedure discussed above has been used extensively in literature [55,56,67,68], it involves approximations, such as use of a straight line $(a+b E)$ to represent the higher $\mathrm{BE}$ region below the pseudogap. So, we have replaced it in the iLf fitting by a free electron-like parabola $\left(a^{\prime} \sqrt{\left(E-b^{\prime}\right)}\right)$ that represents the $s p$ states. However, this is also an approximation, since deviation from parabolic shape would occur since this region has sizeable contribution from the TM $d$ states, besides the Al $s p$ states [32]. Nevertheless, in Fig. S2, we show the results of the fitting by iLf with parabola, where $a^{\prime}$ is varied freely and $b^{\prime}$, which is the bottom 


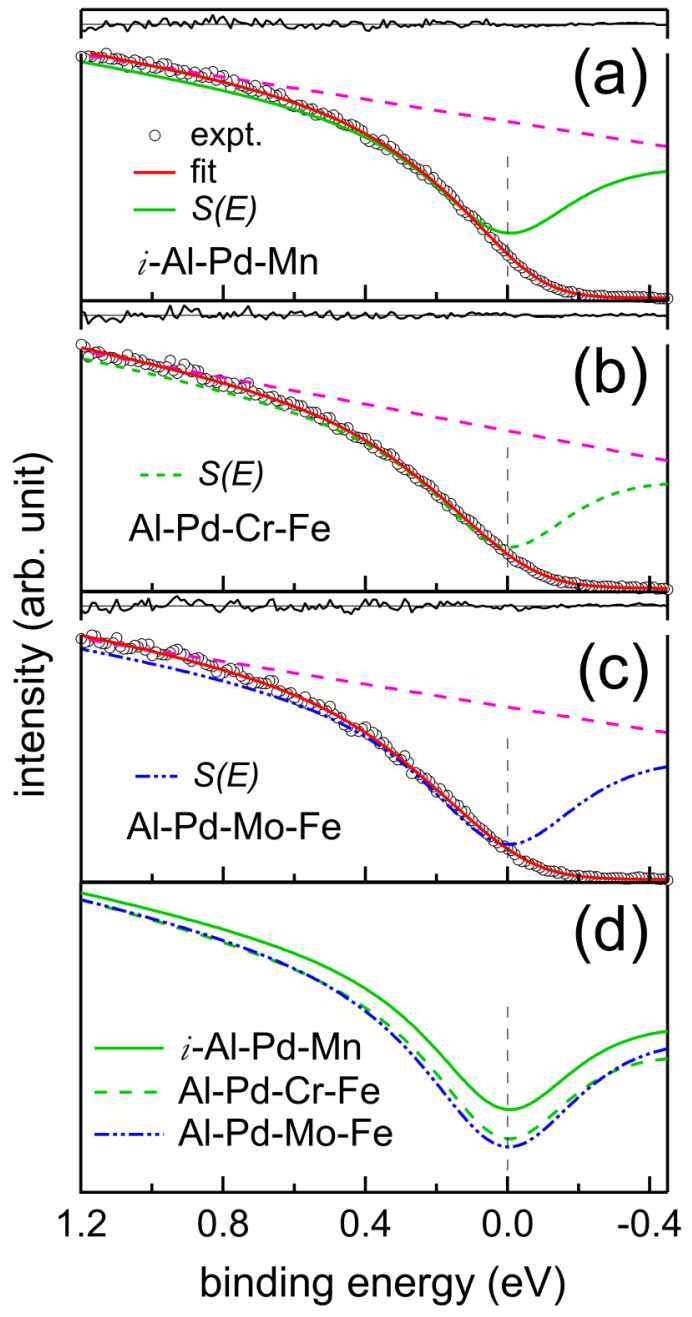

FIG. 2. The near $E_{F}$ region of the VB spectra (black open circles) of (a) $i$-Al-Pd-Mn, (b) Al-Pd-Cr-Fe, and (c) Al-Pd-Mo-Fe fitted using an inverted Lorentzian function $S(E)$ representing the pseudogap, the pink dashed line represents $(a+b E)$. The residual (black curve) is shown at the top of each panel. (d) Comparison of the pseudogaps of Al-Pd-Mo-Fe and Al-Pd-Cr-Fe with $i$-Al-Pd-Mn.

of the $s p$ band, is taken to be $10 \mathrm{eV}$, based on the theoretical $\mathrm{Al}$ $s p$ partial DOS of $i$-Al-Pd-Mn [32]. The quality of the fitting is reasonably good, although the $\chi^{2}$ values are somewhat larger compared to the fitting with straight line (Tables S1-S3). The $C_{L}$ values obtained here also show similar variation between the three compounds.

The standard deviation $(\sigma)$ of the parameters extracted from the fitting such as $C_{L}$ has two components arising from (a) the numerical fitting, and (b) the different ways in which the iLf fitting could be performed, as shown in Tables S1S3. For example, for $C_{L}$ in $i$-Al-Pd-Mn (Al-Pd-Mo-Fe) the numerical $\sigma$ is $0.01(0.01)$, while the $\sigma$ considering the different rows of Table S1 (S3) is $0.017(0.019)$; and thus the total $\sigma$ turns out to be $0.02(0.022)$, these combined $\sigma$ values are shown in Table I. The combined $\sigma$ is significantly smaller than the difference between the $C_{L}$ 's of the three compounds. Moreover, similar variation of $C_{L}$ 's between the three compounds is observed in all cases in Tables S1-S3.

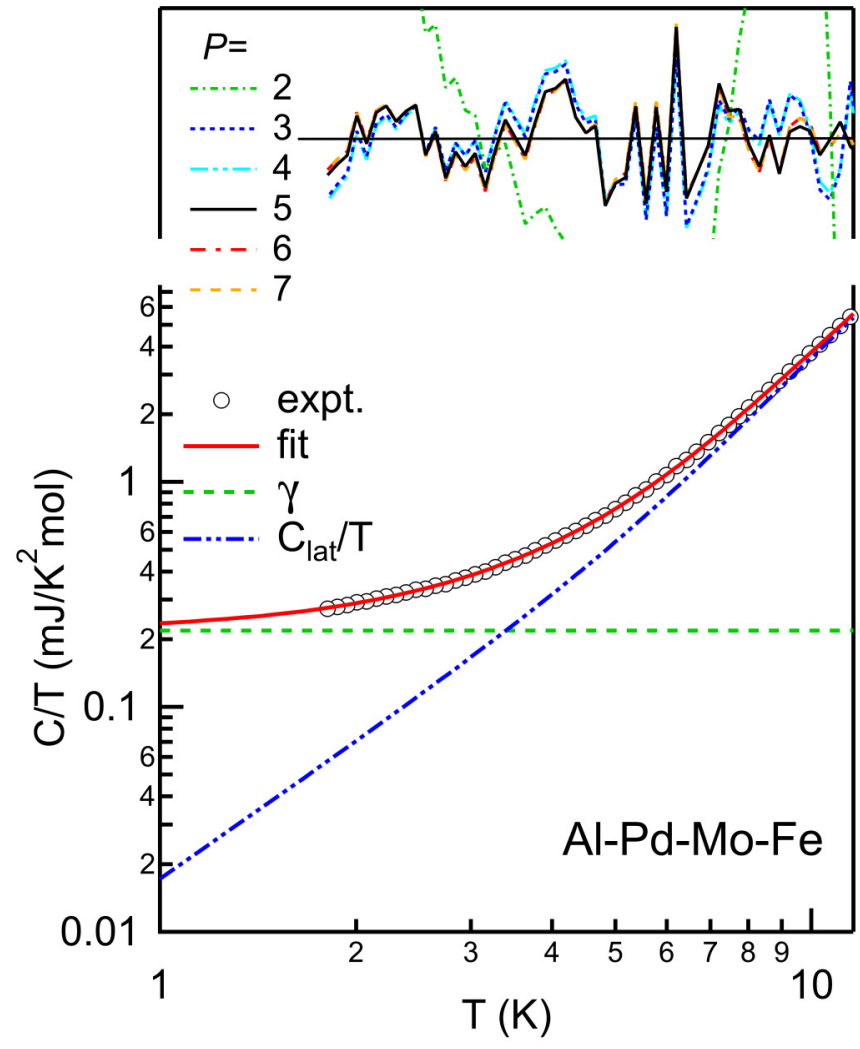

FIG. 3. The specific heat $(C)$ of Al-Pd-Mo-Fe shown as $C / T$ vs $T$ plot along with the fitted curve, $\gamma$ and $C_{\text {lat }} / T$. The residuals of the fit using different $C_{\text {lat }}$ terms $(P)$ are shown in the top panel.

It may be noted that since the use of the iLf function as $S(E)$ is also an approximation, we have performed the fitting using a different function (a third-order polynomial [55]) to represent the pseudogap. Here, $S(E)=C_{0}+$ $C_{1}|E|+C_{2}|E|^{2}+C_{3}|E|^{3}$, where $C_{0}$ determines the intensity at $E_{F}$ with a maximum value of 1 when pseudogap is absent. Thus $\left(1-C_{0}\right)$ can be regarded as a measure of the depth of the pseudogap at $E_{F}$. The other parameters $C_{1}, C_{2}$, and $C_{3}$ determine the shape of the polynomial function. We find that the quality of the fitting is somewhat better in this case compared to iLf, the $\chi^{2}$ being less by about $20 \%$. Here, $\left(1-C_{0}\right)$ increases systematically from $i$-Al-Pd-Mn to Al-Pd-Cr-Fe to Al-Pd-Mo-Fe indicating deepening of the pseudogap, whereas the other parameters are similar (Fig. S3 and Table S4 of Ref. [59]). The above discussions reconfirm that the pseudogap in the high-order approximants studied here is deeper compared to $i$-Al-Pd-Mn.

Deeper pseudogap implies suppression of the electronic density of states at $E_{F}\left[n\left(E_{F}\right)\right]$, and this is also evidenced by the specific heat data of Al-Pd-Mo-Fe. Figure 3 shows the temperature dependence of the specific heat $C$ measured down to $1.8 \mathrm{~K}$. The electronic contribution in the low-temperature limit is given by $\gamma T$, where $\gamma\left(\propto n\left(E_{F}\right)\right)$ is the electronic specific heat coefficient. The primary lattice contribution follows from the usual Debye $T^{3}$-law and is given by $\beta T^{3}$. No upturn in $C / T$ is observed at the lowest temperature indicating negligible magnetic contribution above $1.8 \mathrm{~K}$ in zero magnetic field. 


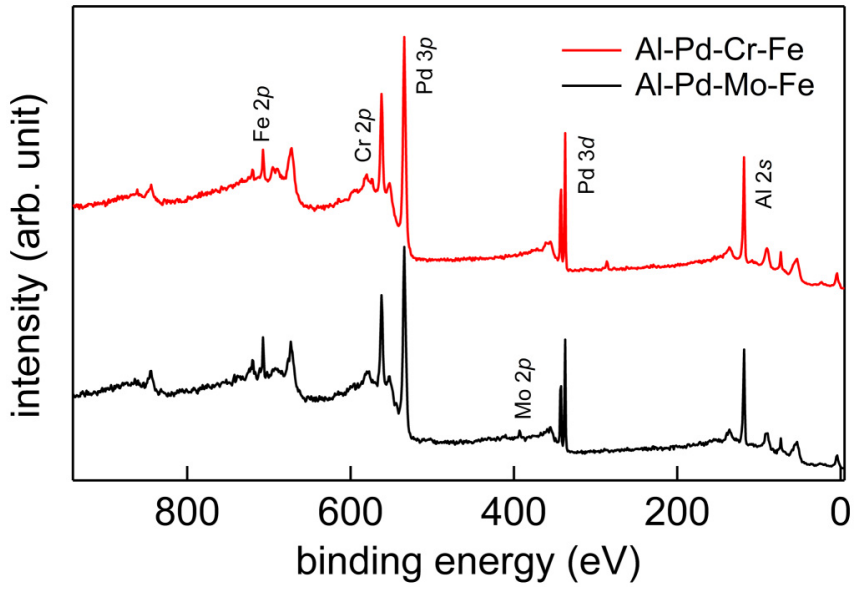

FIG. 4. HAXPES survey spectra of Al-Pd-Cr-Fe and Al-Pd-MoFe with $h v=6 \mathrm{keV}$.

In order to estimate $\gamma$, a nonlinear least-squares fitting is performed using a power series that contains only odd powers of $T[25,70]$,

$$
C=\gamma T+\sum_{n=1}^{P} C_{2 n+1} T^{2 n+1} .
$$

The second term $\left(C_{\text {lat }}\right)$ is related to the lattice contribution, where $C_{3}=\beta$, the other $C_{2 n+1}$ terms are introduced as higherorder corrections, and $P+1$ gives the total number of terms including $\gamma T$. We find that the residual of the fitting shows a large systematic deviation for $P=2$ (top panel in Fig. 3, corresponding fitting not shown). The residual improves substantially for $P=3-4$, but shows further improvement for $P=$ 5 (see in $8-11 \mathrm{~K}$ range), and for larger $P$ it does not improve any more (also the $\chi^{2}$ does not decrease significantly). So, we restrict the fitting to $P=5$ in the temperature range 1.8 to $11.5 \mathrm{~K}$ [25]. The fitting is shown in Fig. 3, where $C_{\text {lat }} / T$ becomes less than $\gamma$ below about $3.5 \mathrm{~K} . \gamma$ turns out to be $0.220 \pm 0.003 \mathrm{~mJ} \mathrm{~K}^{-2} \mathrm{~mol}^{-1}$. The values of all the parameters obtained from the fitting are shown in Table S5 of Ref. [59]. It is important to note that the $\gamma$ for Al-Pd-Mo$\mathrm{Fe}$ is smaller compared to that reported for $i$-Al-Pd-Mn in the literature $\left(0.5-0.25 \mathrm{~mJ} \mathrm{~K}^{-2} \mathrm{~mol}^{-1}[25,71,72]\right)$. As mentioned earlier, since $\gamma$ is proportional to $n\left(E_{F}\right)$, smaller $\gamma$ in Al-Pd-Mo-Fe compared to $i$-Al-Pd-Mn strongly supports the conclusion of deeper pseudogap in the former from HAXPES.

It may also be noted that a correlation between resistivity $(\rho)$ and the depth of the pseudogap obtained from our fitting from $C_{L}$ or $\left(1-C_{0}\right)$ has been observed for different $i$-quasicrystals, as shown in Table S6 in Ref [59]. $\rho$ increases with the depth of the pseudogap in agreement with theoretical expectation [73]. Based on this correlation, it is expected that the resistivity of Al-Pd-Cr-Fe and Al-Pd-Mo-Fe could be larger than $i$-Al-Pd-Mn. The possible reasons for occurrence of pseudogap in the approximants is elucidated in the Discussion section.

Turning to the core level spectra of Al-Pd-Cr-Fe and Al-PdMo-Fe, we first determine their compositions by considering the $\mathrm{Al} 2 s, \mathrm{Pd} 3 p, \mathrm{Cr} 2 p, \mathrm{Fe} 2 p$, and Mo $2 p$ peaks (Fig. 4) and their respective photoemission cross-sections [74]. The

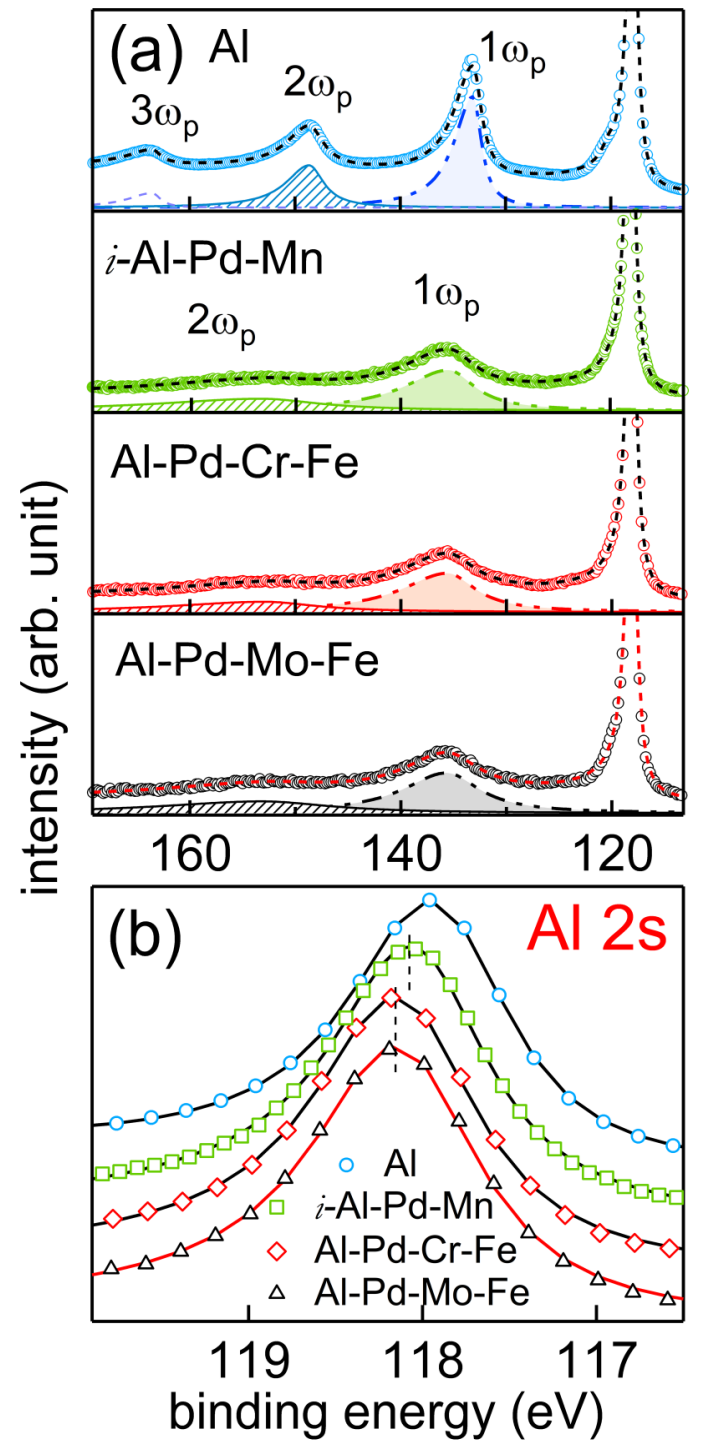

FIG. 5. (a) Al $2 s$ HAXPES $(h v=6 \mathrm{keV})$ core-level spectrum showing the plasmon loss region for $i$-Al-Pd-Mn, Al-Pd-Cr-Fe, Al$\mathrm{Pd}-\mathrm{Mo}-\mathrm{Fe}$, and Al metal with the main peak (shown truncated) normalized to the same intensity. The BE scale for $\mathrm{Al}$ metal is defined $w r t$ its Fermi level position to compensate for the recoil shift [75]. The open circles are the experimental spectra while the dashed curves represent the least-squares curve fits. The plasmon peaks obtained from fitting are shaded. (b) The Al $2 s$ main peak region along with the fitted curves, the spectra have been staggered along the vertical axis. The vertical dashed lines highlight the difference in the peak positions of the approximants and $i$-Al-Pd-Mn.

compositions are $\mathrm{Al}_{69.3} \mathrm{Pd}_{21.4} \mathrm{Cr}_{2.5} \mathrm{Fe}_{6.7}$ for $\mathrm{Al}-\mathrm{Pd}-\mathrm{Cr}-\mathrm{Fe}$ and $\mathrm{Al}_{71.7} \mathrm{Pd}_{20.4} \mathrm{Mo}_{0.7} \mathrm{Fe}_{7.1}$ for $\mathrm{Al}-\mathrm{Pd}-\mathrm{Mo}-\mathrm{Fe}$, which are in good agreement with EPMA. Furthermore, this shows that the $\mathrm{Pd}$ content is slightly less in Al-Pd-Mo-Fe, which in turn explains the lower intensity of the $\mathrm{Pd} 4 d$ related peak in the valence band of Al-Pd-Mo-Fe in Fig. 1(a).

In Fig. 5, the Al $2 s$ spectra of Al-Pd-Cr-Fe and Al-PdMo-Fe have been compared with those of $i$-Al-Pd-Mn and Al metal. A comparison with the multiple bulk plasmon $\left(n \omega_{p}, 1 \omega_{p}=15.4 \mathrm{eV}\right)[62,76]$ peaks for $\mathrm{Al}$ metal (top panel) 


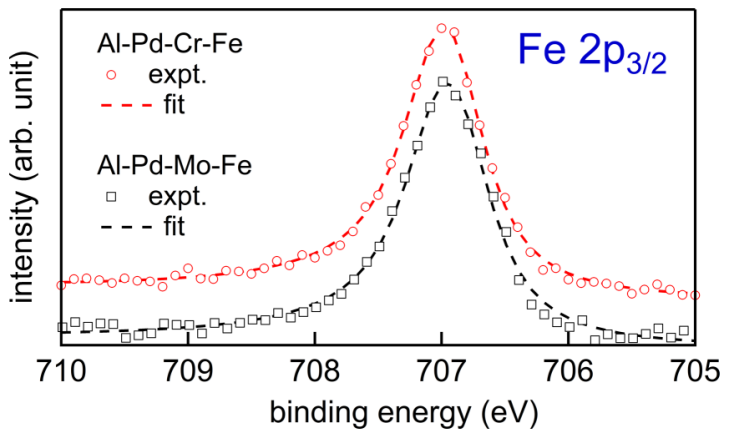

FIG. 6. Fe $2 p_{3 / 2}$ core level spectra of Al-Pd-Cr-Fe and Al-PdMo-Fe along with the fitted curves.

indicates that the broad satellite peaks at similar binding energies in $i$-Al-Pd-Mn, Al-Pd-Cr-Fe, and Al-Pd-Mo-Fe are also related to the plasmons. However, the plasmon frequencies for $i$-Al-Pd-Mn, Al-Pd-Cr-Fe, and Al-Pd-Mo-Fe are similar $\left(\omega_{p}=17.5 \pm 0.1 \mathrm{eV}\right)$, which are larger compared to that of $\mathrm{Al}$ metal. This is related to larger electron density caused by the $\mathrm{TM} d$ electrons, for example, $\mathrm{Mn}(\mathrm{Fe})$ contributes $>5(>6)$ electrons to the valence band compared to 3 in case of Al. Note that this $\omega_{p}$ value for $i$-Al-Pd-Mn is larger than the previous report $(16 \mathrm{eV})$ using XPS that is surface sensitive [60]. This shows the importance of HAXPES, since XPS has a substantial signal from the surface region that has predominant $\mathrm{Al}$ contribution [40,41]. Other evident differences between Al metal and $i$-Al-Pd-Mn or the approximants is the decrease in the intensity and increase in the FWHM of the plasmon peaks in the latter. From the fitting, the relative intensity of $1 \omega_{p}$ with respect to the main peak for $\mathrm{Al}$ metal, $i-\mathrm{Al}-\mathrm{Pd}-\mathrm{Mn}$, $\mathrm{Al}-\mathrm{Pd}-\mathrm{Cr}-\mathrm{Fe}$, and Al-Pd-Mo-Fe is found to be 0.6, 0.52, 0.5, and 0.53 , respectively. On the other hand, their FWHM's show systematic increase: $8.2,8.4$, and $8.8 \mathrm{eV}$ in $i$-Al-Pd-Mn, $\mathrm{Al}-\mathrm{Pd}-\mathrm{Cr}-\mathrm{Fe}$, and $\mathrm{Al}-\mathrm{Pd}-\mathrm{Mo}-\mathrm{Fe}$, respectively. These values are substantially larger than $\mathrm{Al}$ metal $(3.1 \mathrm{eV})$. The reason for the suppression of the plasmon intensities and increased FWHM could be related to $s p$ - $d$ hybridization due to which the $s p$ electron-related plasmon excitation is damped by the $d$ electron interband transitions [77-80].

The Al $2 s$ main peak for both the approximants appears at $118.1 \mathrm{eV}$ [Fig. 5(b)]. Thus these are shifted towards higher binding energy with respect to $i$-Al-Pd-Mn at $118 \mathrm{eV}$. This indicates that the chemical environment, and in particular the Al-TM bonding in the approximants is somewhat different compared to the quasicrystal. From the fitting, we find the intrinsic lifetime widths to be $0.42 \pm 0.005,0.46 \pm 0.002$, $0.47 \pm 0.003$, and $0.49 \pm 0.004$, for Al metal, $i$-Al-Pd-Mn, $\mathrm{Al}-\mathrm{Pd}-\mathrm{Cr}-\mathrm{Fe}$, and Al-Pd-Mo-Fe, respectively. This implies decreasing final state-life time across the series, which could also be associated with the increase of $\mathrm{Al} s p$-TM $d$ hybridization across the series. Thus the changes in the plasmon line shape as well as that of the $\mathrm{Al} 2 s$ main peak (Fig. 5) indicate enhanced $\mathrm{Al} s p$ - TM $d$ hybridization in $\mathrm{Al}-\mathrm{Pd}-\mathrm{Cr}-\mathrm{Fe}$ and $\mathrm{Al}-$ Pd-Mo-Fe compared to $i$-Al-Pd-Mn.

The Fe $2 p_{3 / 2}$ HAXPES spectra for Al-Pd-Cr-Fe and Al-PdMo-Fe appear at similar binding energy of $707 \mathrm{eV}$ (Fig. 6). The FWHM of the $\mathrm{Fe} 2 p_{3 / 2}$ peak of both the approximants are also similar $(\sim 0.6 \mathrm{eV})$, but this value is considerably smaller than that of $\mathrm{Fe}$ metal $(1.06 \mathrm{eV})$ [81]. The larger width in $\mathrm{Fe}$ metal arises from its magnetism that leads to an exchange splitting of about $0.35 \mathrm{eV}$ between adjacent $\mathrm{m}_{j}$ sublevels [81,82]. In case of Al-Pd-Cr-Fe and Al-Pd-Mo-Fe, narrower Fe $2 p_{3 / 2}$ peak and the fact that it can be fitted with a single DS line shape portrays the absence of any exchange splitting, which indicates its nonmagnetic nature.

\section{DISCUSSION}

It has been extensively discussed in the literature that the formation of stable $\mathrm{Al}$ based quasicrystals occurs at certain $e / a$ values [83-86], which indicates that the electronic structure plays an important role in the stability of the icosahedral phases. It is evident that the main constituents ( $\mathrm{Al}$ and $\mathrm{Pd}$ ) are similar between the approximants and the quasicrystal studied here, only $\mathrm{Mn}$ (with outer shell configuration of $3 d^{5} 4 s^{2}$ ) is replaced, in Al-Pd-Cr-Fe by two similar $3 d$ atoms adjacent to it in the periodic table [ $\mathrm{Fe}\left(3 d^{6} 4 s^{2}\right)$ and $\left.\mathrm{Cr}\left(3 d^{4} 4 s^{2}\right)\right]$. Similarly, in case of Al-Pd-Mo-Fe, the two adjacent $d$ atoms are $\mathrm{Fe}\left(3 d^{6} 4 s^{2}\right)$ and Mo $\left(4 d^{4} 5 s^{2}\right)$. In this way, their $e / a$ ratios remain similar to $i$-Al-Pd-Mn. The effective $e / a$ values calculated based on their compositions: $\mathrm{Al}_{69.4} \mathrm{Pd}_{22.3} \mathrm{Cr}_{2.2} \mathrm{Fe}_{6.1}$, $\mathrm{Al}_{70.8} \mathrm{Pd}_{20.3} \mathrm{Mo}_{0.9} \mathrm{Fe}_{7.9}$, and $i-\mathrm{Al}_{70.5} \mathrm{Pd}_{21} \mathrm{Mn}_{8.5}$, are 2.18, 2.23, and 2.22 [84]; and considering the TM $d$ states these are 4.93, 4.84 , and 4.81 , respectively [34]. The similarity of $e / a$ is thus evident, and in particular Al-Pd-Mo-Fe and $i$-Al-Pd-Mn are within $0.5 \%$ of each other.

The existence of pseudogap in Al-Pd-Cr-Fe and Al-Pd-Mo$\mathrm{Fe}$, and that these are deeper compared to $i$-Al-Pd-Mn with all three having nearly similar $e / a$ ratio could possibly be related to the fundamental difference between the translational symmetry of the approximants and the aperiodic order of the quasicrystal. This would suggest that the Hume-Rothery stabilization is more efficient in the high-order approximants with well-defined Fermi surface and BZ in comparison to a quasicrystal. However, other reasons could be at play because, although $\mathrm{Al}-\mathrm{Pd}-\mathrm{Cr}-\mathrm{Fe}$ and $\mathrm{Al}-\mathrm{Pd}-\mathrm{Mo}-\mathrm{Fe}$ are closely related to $i$-Al-Pd-Mn, there are still some differences in their atomic structure, composition and disorder. Both the approximants and $i$-Al-Pd-Mn are most likely comprised of similar kinds of clusters $[47,48]$, but there are discrepancies between the cluster models of the latter and the refined model of Al-Pd-Cr$\mathrm{Fe}$, especially in the way that the clusters are spatially packed. After all, there has been no reliable structural refinement of $i$-Al-Pd-Mn until date. Furthermore, most of the stable icosahedral quasicrystals, except for the binary $\mathrm{Cd}-\mathrm{Yb}$ case, exhibit chemical disorder to some extent [87], and some intrinsic disorder known as phason disorder. Phason disorder is found to be absent in the approximants, but possible chemical as well as positional disorder in the $M$ clusters have been indicated, although a full characterization has not been performed [45].

The differences in their atomic structure and composition might manifest in the electronic structure as stronger hybridization between $\mathrm{Al} s p$ and TM $d$ states in the approximants compared to the quasicrystal. This is indicated by the core-level spectra discussed earlier (Fig. 5), which also shows that the hybridization is strongest in Al-Pd-Mo-Fe. This is possibly caused by presence of the $4 d$ element Mo, as well 
as due to Pd deficient and $\mathrm{Fe}$ excess composition of $\mathrm{Al}-$ $\mathrm{Pd}-\mathrm{Mo}-\mathrm{Fe}$ compared to Al-Pd-Cr-Fe. Atomic rearrangement could also occur with Mo occupying Pd positions because of their similar atomic radius. DFT studies have established that Al $s p$ - TM $d$ hybridization enhances the pseudogap in Al-TM systems and the pseudogap is more pronounced in $s p d$ compounds compared to $s p$ compounds $[34,39,88]$. It was also shown that substitution of $3 d$ TM's (Mn, Co) by $4 d$ TM's (Tc, $\mathrm{Rh}$ ) enhances the width of the band gap that forms due to $\mathrm{Al}$ $s p$ - TM $d$ hybridization $[34,89,90]$.

Here, we address the possibility of a rigid band shift, i.e., a shift of the electronic bands due to a change in electron occupancy, with the band structure otherwise remaining unchanged. This would cause a constant shift in the DOS features, but the spectra in Fig. 1(a) do not show any significant shift that would have been readily visible from the $\mathrm{Pd} d$ peak and the region on the lower BE side of this peak. So, we do not find any evidence of rigid band shift from our data. However, change in the position of the pseudogap minimum without any change in the $\mathrm{Pd} d$ peak (i.e., a nonrigid bandlike modification of DOS) cannot be ruled out, since this depends on the details of the atomic arrangement that influences their electronic structure, as shown recently for a related quasicrystal $i$-Al-Pd-Re [91]. In case of $i$-Al-Pd-Mn, however, the DFT calculations [32] show that the pseudogap minimum is at $E_{F}$ for the high order $8 / 5$ approximant. To examine this from our fitting, we have allowed the position of the minimum to vary. We find that the shift is insignificant for $i$-Al-Pd-Mn $(0.004 \mathrm{eV})$, justifying our earlier proposition (Fig. 2 and Table I) and that in literature $[32,55,68]$. In contrast to $i$-Al-Pd-Mn, the pseudogap shifts slightly above $E_{F}$ to $-0.04 \mathrm{eV}$ for Al-Pd-Mo-Fe, which is opposite of what could be expected from its marginally larger $e / a$. Thus the observed shifts cannot be explained simply by $e / a$, as these depend on the details of their electronic structure, and in particular on the shape and intensity of the different states ( $\mathrm{Al} s p, \mathrm{TM} d)$ in the near $E_{F}$ region. The pseudogap appears slightly below $E_{F}$ at $0.04 \mathrm{eV}$ for Al-Pd-Cr-Fe. It is important to note that despite the shift in the pseudogap minimum, the trend in the variation of the depth of the pseudogap in the three compounds remains unchanged, and in fact $C_{L}(=0.86)$ becomes somewhat larger in Al-Pd-Mo-Fe, whereas it decreases slightly in Al-Pd-CrFe (compare Table I and Table S7 in Ref. [59]).

While we have discussed above some plausible reasons for our experimental observations from HAXPES and specific heat data, a definitive statement would require a detailed theoretical study based on realistic structure of $i$-Al-Pd-Mn and the approximants. But, the impediment is that the structure of $i$-Al-Pd-Mn is not known, and even if it was known, disorder would be very difficult to handle within the DFT formalism. However, the structure of Al-Pd-Cr-Fe has been determined [45], and although future ab initio pseudopotential calculation might throw some light on the origin of their pseudogap, the reason why these are deeper than $i$-Al-Pd-Mn will require the atomic structure of the latter to be determined. Furthermore, even for simplified model structures that could be constructed to represent the quasicrystal and the approximants, using a few tiling units with appropriate atomic decorations (e.g., decorated Penrose rhombi to represent two-dimensional structures), the difficulty is to calculate the electronic structure of a quasicrystal in a truly aperiodic formalism, which to the best of our knowledge has not been developed until date in the framework of band theory.

\section{CONCLUSIONS}

We report the results of our experimental investigation on the bulk electronic structure of high-order approximants (Al-Pd-Cr-Fe and Al-Pd-Mo-Fe) using HAXPES that is compared to a closely related $i$-Al-Pd-Mn quasicrystal with nearly identical $e / a$ ratios. The near $E_{F}$ valence band spectra of both the approximants show the existence of a well-formed pseudogap at $E_{F}$ and surprisingly it is deeper compared to $i$-Al-Pd-Mn. The latter is reconfirmed by smaller value of the coefficient of the electronic contribution to the specific heat $(\gamma)$ in Al-Pd-Mo-Fe compared to $i$-Al-Pd-Mn.

The suppression of the plasmon intensities, and increase in the width as well as lifetime broadening of the $\mathrm{Al} 2 \mathrm{~s}$ main peak in Al-Pd-Cr-Fe and Al-Pd-Mo-Fe compared to $i$-Al-Pd-Mn point to enhanced Al $s p$ - TM $d$ hybridization in the approximants. Thus besides the fundamental difference in their periodicities, $s p-d$ hybridization might play a significant role in formation of the pseudogap in high-order approximants comparable to (even deeper, as in this case) a related quasicrystal. Moreover, since the resistivity of the quasicrystals increases with the depth of the pseudogap, the occurrence of pseudogap in both high-order approximants and a quasicrystal predicts the similarity of their transport properties. The $\mathrm{Fe}$ $2 p$ core level spectra of the approximants have a markedly smaller width compared to the Fe metal due to the absence of magnetic exchange splitting, indicating nonmagnetic nature of the former.

Our work provides a pathway in the search for new quasicrystalline phases. If the depth and the shape of the pseudogap of a newly identified approximant phase is comparable to the quasicrystals, a quasicrystalline phase could be expected nearby in the phase space. Our work suggests that high-order approximants are not merely geometrical interpolation between simpler low-order approximants and quasicrystals, but something that could possibly shed new light in future on the stabilization mechanisms of related quasicrystals and complex metallic alloys. It is our hope that the present study will stimulate further research in the electronic structure calculations for aperiodic systems, and also induce renewed vigor in unraveling their atomic structure.

\section{ACKNOWLEDGMENTS}

We are grateful to M. Krajčí for stimulating discussions, a careful reading of the manuscript, and sharing some results of his calculation that enriched our understanding. We are thankful to M. Mihalkovic for introducing us to this research topic and providing valuable suggestions. The HAXPES experiments were carried out at PETRA III of Deutsches Elektronen-Synchrotron, a member of Helmholtz-Gemeinschaft Deutscher Forschungszentren. Financial support by the D.S.T., Government of India within the framework of India@DESY collaboration is gratefully acknowledged. This work was also supported in part by JSPS 
KAKENHI Grants No. JP19H05819 and No. JP19H05821. The high-resolution powder XRD data were collected at the BL15XU beamline of SPring-8 under the approval of National Institute for Materials Science (NIMS, Tsukuba, Japan) (Proposal No. 2017A4901). We thank Ph. Ebert and K. Horn for providing the Al-Pd-Mn sample for HAXPES measurement. C. Schlueter, W. Drube, C. Narayana, and M. Sanyal are thanked for support and encouragement. We would like to acknowledge skilful technical support from K. Ederer and I. Schostak.
[1] A. I. Goldman and R. F. Kelton, Quasicrystals and crystalline approximants, Rev. Mod. Phys. 65, 213 (1993).

[2] D. Levine and P. J. Steinhardt, Quasicrystals: A New Class of Ordered Structures, Phys. Rev. Lett. 53, 2477 (1984).

[3] D. Shechtman, I. Blech, D. Gratias, and J. W. Cahn, Metallic Phase with Long-Range Orientational Order and no Translational Symmetry, Phys. Rev. Lett. 53, 1951 (1984).

[4] A. P. Tsai, J. Q. Guo, E. Abe, H. Takakura, and T. J. Sato, A stable binary quasicrystal, Nature 408, 537 (2000).

[5] S. Förster, K. Meinel, R. Hammer, M. Trautmann, and W. Widdra, Quasicrystalline structure formation in a classical crystalline thin-film system, Nature 502, 215 (2013).

[6] S. Förster, M. Trautmann, S. Roy, W. A. Adeagbo, E. M. Zollner, R. Hammer, F. O. Schumann, K. Meinel, S. K. Nayak, K. Mohseni, W. Hergert, H. L. Meyerheim, and W. Widdra, Observation and Structure Determination of an Oxide Quasicrystal Approximant, Phys. Rev. Lett. 117, 095501 (2016).

[7] D. V. Talapin, E. V. Shevchenko, M. I. Bodnarchuk, X. Ye, J. Chen, and C. B. Murray, Quasicrystalline order in selfassembled binary nanoparticle superlattices, Nature 461, 964 (2009).

[8] V. K. Singh, M. Mihalkovic, M. Krajčí, S. Sarkar, P. Sadhukhan, M. Maniraj, A. Rai, K. Pussi, D. L. Schlagel, T. A. Lograsso, A. K. Shukla, and S. R. Barman, Quasiperiodic ordering in thick Sn layer on $i$-Al-Pd-Mn: A possible quasicrystalline clathrate, Phys. Rev. Research 2, 013023 (2020).

[9] C. R. Iacovella, A. S. Keys, and S. C. Glotzer, Self-assembly of soft-matter quasicrystals and their approximants, Proc. Natl. Acad. Sci. U.S.A. 108, 20935 (2011).

[10] K. Deguchi, S. Matsukawa, N. K. Sato, T. Hattori, K. Ishida, H. Takakura, and T. Ishimasa, Quantum critical state in a magnetic quasicrystal, Nat. Mater. 11, 1013 (2012).

[11] K. Kamiya, T. Takeuchi, N. Kabeya, N. Wada, T. Ishimasa, A. Ochiai, K. Deguchi, K. Imura, and N. K. Sato, Discovery of superconductivity in quasicrystal, Nat. Commun. 9, 154 (2018).

[12] S. Sakai and R. Arita, Exotic pairing state in quasicrystalline superconductors under a magnetic field, Phys. Rev. Res. 1, 022002(R) (2019).

[13] Y. E. Kraus and O. Zilberberg, Quasiperiodicity and topology transcend dimensions, Nat. Phys. 12, 624 (2016).

[14] H. Huang and F. Liu, Quantum Spin Hall Effect and Spin Bott Index in a Quasicrystal Lattice, Phys. Rev. Lett. 121, 126401 (2018).

[15] D. Varjas, A. Lau, K. Pöyhönen, A. R. Akhmerov, D. I. Pikulin, and I. C. Fulga, Topological Phases without Crystalline Counterparts, Phys. Rev. Lett. 123, 196401 (2019).

[16] K. Inagaki, S. Suzuki, A. Ishikawa, T. Tsugawa, F. Aya, T. Yamada, K. Tokiwa, T. Takeuchi, and R. Tamura, Ferromagnetic 2/1 quasicrystal approximants, Phys. Rev. B 101, 180405(R) (2020).
[17] S. Yoshida, S. Suzuki, T. Yamada, T. Fujii, A. Ishikawa, and R. Tamura, Antiferromagnetic order survives in the higher-order quasicrystal approximant, Phys. Rev. B 100, 180409(R) (2019).

[18] Y. G. So, K. Takagi, and T. J. Sato, Formation and magnetic properties of $\mathrm{Ga}-\mathrm{Pd}-\mathrm{Tb} 2 / 1$ approximant, J. Phys.: Conf. Ser. 1458, 012003 (2020).

[19] M. Maniraj, A. Rai, S. R. Barman, M. Krajčí, D. L. Schlagel, T. A. Lograsso, and K. Horn, Unoccupied electronic states of icosahedral Al-Pd-Mn quasicrystals: Evidence of image potential resonance and pseudogap, Phys. Rev. B 90, 115407 (2014).

[20] V. Smetana, S. P. Kelley, A.-V. Mudring, and R. D. Rogers, A fivefold $\mathrm{UO}_{2}^{2+}$ node is a path to dodecagonal quasicrystal approximants in coordination polymers, Sci. Adv. 6, eaay7685 (2020).

[21] C. Dong, The concept of the approximants of quasicrystals, Scr. Metall. Mater. 33, 239 (1995).

[22] S. Poon, Electronic properties of quasicrystals an experimental review, Adv. Phys. 41, 303 (1992).

[23] T. Takeuchi and U. Mizutani, Electronic structure, electron transport properties, and relative stability of icosahedral quasicrystals and their $1 / 1$ and 2/1 approximants in the Al-Mg-Zn alloy system, Phys. Rev. B 52, 9300 (1995).

[24] T. Shibuya, K. Edagawa, and S. Takeuchi, Proceedings of the 6th International Conference on Quasicrystals (World Scientific, Singapore, 1998), p. 700.

[25] C. A. Swenson, I. R. Fisher, N. E. Anderson, P. C. Canfield, and A. Migliori, Icosahedral quasicrystal $\mathrm{Al}_{71} \mathrm{Pd}_{21} \mathrm{Mn}_{08}$ and its $\xi^{\prime}$ approximant: Linear expansivity, specific heat, magnetic susceptibility, electrical resistivity, and elastic constants, Phys. Rev. B 65, 184206 (2002).

[26] N. Koshikawa, S. Yoda, K. Edagawa, K. Minoda, R. Tamura, and $\mathrm{S}$. Takeuchi, Electrical resistivity of the $\mathrm{Al}_{65} \mathrm{Rh}_{27} \mathrm{Si}_{8} 2 / 1$ cubic approximant, J. Non-Cryst. Solids 334-335, 372 (2004).

[27] J.-M. Dubois, Properties- and applications of quasicrystals and complex metallic alloys, Chem. Soc. Rev. 41, 6760 (2012).

[28] J. Hasegawa, R. Tamura, and S. Takeuchi, Electronic transport of the icosahedral $\mathrm{Zn}-\mathrm{Mg}$-Sc quasicrystal and its cubic approximant $\mathrm{Zn}_{17} \mathrm{Sc}_{3}$, Phys. Rev. B 66, 132201 (2002).

[29] T. Fujiwara and T. Yokokawa, Universal Pseudogap at Fermi Energy in Quasicrystals, Phys. Rev. Lett. 66, 333 (1991).

[30] J. Hafner and M. Krajčí, Electronic Structure and Stability of Quasicrystals: Quasiperiodic Dispersion Relations and Pseudogaps, Phys. Rev. Lett. 68, 2321 (1992).

[31] J. Hafner and M. Krajcí, Electronic structure of rational approximants to icosahedral quasicrystals, Europhys. Lett. 17, 145 (1992).

[32] M. Krajčí, M. Windisch, J. Hafner, G. Kresse, and M. Mihalkovič, Atomic and electronic structure of icosahedral AlPd-Mn alloys and approximant phases, Phys. Rev. B 51, 17355 (1995). 
[33] S. Roche and T. Fujiwara, Fermi surfaces and anomalous transport in quasicrystals, Phys. Rev. B 58, 11338 (1998).

[34] M. Krajčí and J. Hafner, Semiconducting Al-transition-metal quasicrystals, Phys. Rev. B 68, 165202 (2003).

[35] B. Frigan, A. Santana, M. Engel, D. Schopf, H.-R. Trebin, and M. Mihalkovič, Low-temperature structure of $\xi^{\prime}$-Al-Pd-Mn optimized by ab initio methods, Phys. Rev. B 84, 184203 (2011).

[36] A.-P. Tsai, A test of Hume-Rothery rules for stable quasicrystals, J. Non-Cryst. Solids 334-335, 317 (2004).

[37] A. P. Tsai, A. Inoue, Y. Yokoyama, and T. Masumoto, Stable icosahedral Al-Pd-Mn and Al-Pd-Re alloys, Mater. Trans., JIM 31, 98 (1990).

[38] R. Tamura, Y. Murao, S. Takeuchi, T. Kiss, T. Yokoya, and S. Shin, Comparative study of the binary icosahedral quasicrystal $\mathrm{Cd}_{5.7} \mathrm{Yb}$ and its crystalline approximant $\mathrm{Cd}_{6} \mathrm{Yb}$ by low-temperature ultrahigh-resolution photoemission spectroscopy, Phys. Rev. B 65, 224207 (2002).

[39] Y. Ishii and T. Fujiwara, Hybridization Mechanism for Cohesion of Cd-Based Quasicrystals, Phys. Rev. Lett. 87, 206408 (2001).

[40] M. Gierer, M. A. van Hove, A. I. Goldman, Z. Shen, S.-L. Chang, C. J. Jenks, C.-M. Zhang, and P. A. Thiel, Structural Analysis of the Fivefold Symmetric Surface of the $\mathrm{Al}_{70} \mathrm{Pd}_{21} \mathrm{Mn}_{9}$ Quasicrystal by Low Energy Electron Diffraction, Phys. Rev. Lett. 78, 467 (1997).

[41] G. Neuhold, S. R. Barman, K. Horn, W. Theis, P. Ebert, and K. Urban, Enhanced surface metallic density of states in icosahedral quasicrystals, Phys. Rev. B 58, 734 (1998).

[42] C. H. Chen, D. C. Joy, H. S. Chen, and J. J. Hauser, Observation of Anomalous Plasmon Linewidth in the Icosahedral Al-Mn Quasicrystals, Phys. Rev. Lett. 57, 743 (1986).

[43] M. Terauchi, M. Tanaka, A.-P. Tsai, A. Inoue, and T. Masumoto, Electron-energy-loss spectroscopy study of the electronic structure of a stable quasicrystal $\mathrm{Al}_{65} \mathrm{Cu}_{20} \mathrm{Ru}_{15}$, Philos. Mag. Lett. 74, 107 (1996).

[44] K. Kirihara, T. Nakata, M. Takata, Y. Kubota, E. Nishibori, K. Kimura, and M. Sakata, Covalent Bonds in AlMnSi Icosahedral Quasicrystalline Approximant, Phys. Rev. Lett. 85, 3468 (2000).

[45] N. Fujita, H. Takano, A. Yamamoto, and A.-P. Tsai, Clusterpacking geometry for Al-based F-type icosahedral alloys, Acta Crystallogr. Sect. A 69, 322 (2013).

[46] N. Fujita and M. Ogashiwa, A unified geometrical framework for face-centered icosahedral approximants in Al-Pd-TM ( $\mathrm{TM}=$ transition metal) systems, Mater. Trans. (2021), doi: 10.2320/matertrans.MT-MB2020007.

[47] A. Katz and D. Gratias, A geometric approach to chemical ordering in icosahedral structures, J. Non-Cryst. Solids 153-154, 187 (1993).

[48] V. Elser, Random tiling structure of icosahedral quasicrystals, Philos. Mag. B 73, 641 (1996).

[49] Hard X-ray Photoelectron Spectroscopy (HAXPES), edited by J. Woicik (Springer International, Switzerland, 2016).

[50] A. X. Gray, C. Papp, S. Ueda, B. Balke, Y. Yamashita, L. Plucinski, J. Minár, J. Braun, E. R. Ylvisaker, C. M. Schneider, W. E. Pickett, H. Ebert, K. Kobayashi, and C. S. Fadley, Probing bulk electronic structure with hard X-ray angle-resolved photoemission, Nat. Mater. 10, 759 (2011).

[51] A. X. Gray, J. Minár, S. Ueda, P. R. Stone, Y. Yamashita, J. Fujii, J. Braun, L. Plucinski, C. M. Schneider, G. Panaccione,
H. Ebert, O. D. Dubon, K. Kobayashi, and C. S. Fadley, Bulk electronic structure of the dilute magnetic semiconductor $\mathrm{Ga}_{1-x} \mathrm{Mn}_{x}$ As through hard X-ray angle-resolved photoemission, Nat. Mater. 11, 957 (2012).

[52] J. Fujii, B. R. Salles, M. Sperl, S. Ueda, M. Kobata, K. Kobayashi, Y. Yamashita, P. Torelli, M. Utz, C. S. Fadley, A. X. Gray, J. Braun, H. Ebert, I. Di Marco, O. Eriksson, P. Thunström, G. H. Fecher, H. Stryhanyuk, E. Ikenaga, J. Minár, C. H. Back, G. van der Laan, and G. Panaccione, Identifying the Electronic Character and Role of the Mn States in the Valence Band of (Ga,Mn)As, Phys. Rev. Lett. 111, 097201 (2013).

[53] M. Horio, Y. Krockenberger, K. Yamamoto, Y. Yokoyama, K. Takubo, Y. Hirata, S. Sakamoto, K. Koshiishi, A. Yasui, E. Ikenaga, S. Shin, H. Yamamoto, H. Wadati, and A. Fujimori, Electronic Structure of Ce-Doped and -Undoped $\mathrm{nd}_{2} \mathrm{CuO}_{4}$ Superconducting Thin Films Studied by Hard X-Ray Photoemission and Soft X-Ray Absorption Spectroscopy, Phys. Rev. Lett. 120, 257001 (2018).

[54] P. Sadhukhan, S. W. D’Souza, V. K. Singh, R. S. Dhaka, A. Gloskovskii, S. K. Dhar, P. Raychaudhuri, A. Chainani, A. Chakrabarti, and S. Roy Barman, Role of antisite disorder, electron-electron correlations, and a surface valence transition in the electronic structure of $\mathrm{CeMnNi}_{4}$, Phys. Rev. B 99, 035102 (2019).

[55] J. Nayak, M. Maniraj, A. Rai, S. Singh, P. Rajput, A Gloskovskii, J. Zegenhagen, D. L. Schlagel, T. A. Lograsso, K. Horn, and S. R. Barman, Bulk Electronic Structure of Quasicrystals, Phys. Rev. Lett. 109, 216403 (2012).

[56] J. Nayak, M. Maniraj, A. Gloskovskii, M. Krajčí, S. Sebastian, I. R. Fisher, K. Horn, and S. R. Barman, Bulk electronic structure of Zn-Mg-Y and Zn-Mg-Dy icosahedral quasicrystals, Phys. Rev. B 91, 235116 (2015).

[57] J. Strempfer, S. Francoual, D. Reuther, D. K. Shukla, A. Skaugen, H. Schulte-Schrepping, T. Kracht, and H. Franz, Resonant scattering and diffraction beamline P09 at PETRA III, J. Synchrotron Radiat. 20, 541 (2013).

[58] A. Gloskovskii, G. Stryganyuk, G. H. Fecher, C. Felser, S. Thiess, H. Schulz-Ritter, W. Drube, G. Berner, M. Sing, R. Claessen, and M. Yamamoto, Magnetometry of buried layersLinear magnetic dichroism and spin detection in angular resolved hard X-ray photoelectron spectroscopy, J. Electron Spectrosc. Relat. Phenom. 185, 47 (2012).

[59] See Supplemental Material at http://link.aps.org/supplemental/ 10.1103/PhysRevResearch.3.013151 for Figs. S1-S3 and Table S1-S7.

[60] A. K. Shukla, R. S. Dhaka, C. Biswas, S. Banik, S. R. Barman, K. Horn, P. Ebert, and K. Urban, Growth and electronic structure of alkali-metal adlayers on icosahedral $\mathrm{Al}_{70.5} \mathrm{Pd}_{21} \mathrm{Mn}_{8.5}$, Phys. Rev. B 73, 054432 (2006).

[61] S. Doniach and M. Sunjic, Many-electron singularity in X-ray photoemission and X-ray line spectra from metals, J. Phys. C 3, 285 (1970).

[62] C. Biswas, A. K. Shukla, S. Banik, V. K. Ahire, and S. R. Barman, Plasmons in core-level photoemission spectra of Al(111), Phys. Rev. B 67, 165416 (2003).

[63] S. Tougaard, Practical algorithm for background subtraction, Surf. Sci. 216, 343 (1989).

[64] C. Biswas, A. K. Shukla, S. Banik, S. R. Barman, and A. Chakrabarti, Argon Nanobubbles in $\mathrm{Al}(111)$ : A Photoemission Study, Phys. Rev. Lett. 92, 115506 (2004). 
[65] C. Fadley, X-ray photoelectron spectroscopy: Progress and perspectives, J. Electron Spectrosc. Relat. Phenom. 178-179, 2 (2010).

[66] A. K. Shukla, R. S. Dhaka, S. W. D'Souza, M. Maniraj, S. R. Barman, K. Horn, P. Ebert, K. Urban, D. Wu, and T. A. Lograsso, Manganese adlayers on i-Al-Pd-Mn quasicrystal: growth and electronic structure, J. Phys.: Condens. Matter 21, 405005 (2009).

[67] M. Mori, S. Matsuo, T. Ishimasa, T. Matsuura, K. Kamiya, H. Inokuchi, and T. Matsukawa, Photoemission study of an Al-CuFe icosahedral phase, J. Phys.: Condens. Matter 3, 767 (1991).

[68] Z. M. Stadnik, D. Purdie, M. Garnier, Y. Baer, A. P. Tsai, A. Inoue, K. Edagawa, and S. Takeuchi, Electronic Structure of Icosahedral Alloys Studied by Ultrahigh Energy Resolution Photoemission Spectroscopy, Phys. Rev. Lett. 77, 1777 (1996).

[69] This value is about $10 \%$ smaller compared to Ref. [55] $\left(\mathrm{C}_{L}=\right.$ $0.72)$. This is attributed to higher photon energy $(h \nu=8 \mathrm{keV})$ used resulting in increased inelastic mean free path as well as variation in photoemission cross-section.

[70] T. Barron, J. Collins, and G. White, Thermal expansion of solids at low temperatures, Adv. Phys. 29, 609 (1980).

[71] M. A. Chernikov, A. Bernasconi, C. Beeli, A. Schilling, and H. R. Ott, Low-temperature magnetism in icosahedral $\mathrm{Al}_{70} \mathrm{Mn}_{9} \mathrm{Pd}_{21}$, Phys. Rev. B 48, 3058 (1993).

[72] J. C. Lasjaunias, A. Sulpice, N. Keller, J. J. Préjean, and M. de Boissieu, Magnetic and calorimetric study of a single grain of quasicrystalline Al-Pd-Mn, Phys. Rev. B 52, 886 (1995).

[73] U. Mizutani, Role of the pseudogap in the electron transport of quasicrystals and their approximants, J. Phys.: Condens. Matter 10, 4609 (1998).

[74] M. Trzhaskovskaya and V. Yarzhemsky, Dirac-Fock photoionization parameters for HAXPES applications, At. Data Nucl. Data Tables 119, 99 (2018).

[75] Y. Takata, Y. Kayanuma, S. Oshima, S. Tanaka, M. Yabashi, K. Tamasaku, Y. Nishino, M. Matsunami, R. Eguchi, A. Chainani, M. Oura, T. Takeuchi, Y. Senba, H. Ohashi, S. Shin, and T. Ishikawa, Recoil Effect of Photoelectrons in the Fermi Edge of Simple Metals, Phys. Rev. Lett. 101, 137601 (2008).

[76] R. S. Dhaka and S. R. Barman, Plasmon Excitations by Photoelectron Emission from Rare Gas Nanobubbles in Aluminum, Phys. Rev. Lett. 104, 036803 (2010).

[77] T. Grenet and M. Cheynet, Plasmons in icosahedral quasicrystals: An EELS investigation, Eur. Phys. J. B 13, 701 (2000).

[78] M. Zurkirch, M. D. Crescenzi, M. Erbudak, M. Hochstrasser, and A. R. Kortan, Comparison of the electronic structure of AlPd and $\mathrm{Al}_{70} \mathrm{Pd}_{20} \mathrm{Mn}_{10}$, Phys. Rev. B 55, 8808 (1997).
[79] P. Sadhukhan, S. Barman, T. Roy, V. K. Singh, S. Sarkar, A. Chakrabarti, and S. R. Barman, Electronic structure of AuSn compounds grown on Au(111), Phys. Rev. B 100, 235404 (2019).

[80] P. Sadhukhan, D. Pandey, V. K. Singh, S. Sarkar, A. Rai, K. Bhattacharya, A. Chakrabarti, and S. R. Barman, Electronic structure and morphology of thin surface alloy layers formed by deposition of Sn on Au(111), Appl. Surf. Sci. 506, 144606 (2020).

[81] G. Rossi, G. Panaccione, F. Sirotti, S. Lizzit, A. Baraldi, and G. Paolucci, Magnetic dichroism in the angular distribution of Fe $2 p$ and $3 p$ photoelectrons: Empirical support to Zeeman-like analysis, Phys. Rev. B 55, 11488 (1997).

[82] L. Baumgarten, C. M. Schneider, H. Petersen, F. Schäfers, and J. Kirschner, Magnetic X-Ray Dichroism in Core-Level Photoemission from Ferromagnets, Phys. Rev. Lett. 65, 492 (1990).

[83] A.-P. Tsai, Discovery of stable icosahedral quasicrystals: progress in understanding structure and properties, Chem. Soc. Rev. 42, 5352 (2013).

[84] U. Mizutani, H. Sato, M. Inukai, and E. Zijlstra, e/a determination for $4 \mathrm{~d}$ - and 5d-transition metal elements and their intermetallic compounds with $\mathrm{Mg}, \mathrm{Al}, \mathrm{Zn}, \mathrm{Cd}$ and $\mathrm{In}$, Philos. Mag. 93, 3353 (2013).

[85] A. P. Tsai, Metallurgy of quasicrystals, in Physical Properties of Quasicrystals, Springer Series in Solid-State Sciences, edited by Z. M. Stadnik, Vol. 126 (Springer, Berlin, Heidelberg, 1999), pp. 5-50.

[86] U. Mizutani, M. Inukai, H. Sato, and E. S. Zijlstra, HumeRothery stabilization mechanism and e/a determination for RTand MI-type 1/1-1/1-1/1 approximants studied by FLAPWFourier analyses, Chem. Soc. Rev. 41, 6799 (2012).

[87] M. de Boissieu, P. Stephens, M. Boudard, C. Janot, D. L. Chapman, and M. Audier, Disorder and Complexity in the Atomic Structure of the Perfect Icosahedral Alloy of Al-Pd-Mn, Phys. Rev. Lett. 72, 3538 (1994).

[88] G. T. de Laissardière, D. Nguyen-Manh, and D. Mayou, Electronic structure of complex Hume-Rothery phases and quasicrystals in transition metal aluminides, Prog. Mater. Sci. 50, 679 (2005).

[89] M. Krajčí and J. Hafner, Covalent bonding and band-gap formation in ternary transition-metal di-aluminides: $\mathrm{Al}_{4} \mathrm{MnCo}$ and related compounds, J. Phys.: Condens. Matter 14, 7201 (2002).

[90] M. Krajcí and J. Hafner, Covalent bonding and bandgap formation in transition-metal aluminides: di-aluminides of group VIII transition metals, J. Phys.: Condens. Matter 14, 5755 (2002).

[91] S. Sarkar et al. (unpublished). 